\title{
Successful Recanalization by Catheter-Directed Thrombolysis with a Continuous Intra-arterial Infusion of Low-Dose Tirofiban for Acute Carotid In-Stent Thrombosis
}

\author{
Yang Heping ${ }^{1}$, Zhang Guiqiang ${ }^{1}$
}

\begin{abstract}
${ }^{1}$ Department of Neurology, the Guangxi Zhuang Autonomous Region nationalities hospital, Nanning, Guangxi, China.Code:530000

Abstract: A 65-year-old female presented with severe left cervical internal carotid artery stenosis manifesting as repeated transient ischemic attacks(TIAs) consisting of right hemiparesis and motor aphasia. Carotid artery stenting (CAS) under distal protection was performed to prevent further ischemic events. This procedure was uneventful. However, the patient exhibited progressive right hemiparesis and motor aphasia three hours after CAS. Emergent angiography revealed carotid artery occlusion due to acute carotid in-stent thrombosis (ACIST). Mechanical thrombectomy was performed. The carotid artery was recanalized with small residual thrombus. The neurological deficits partial disappeared after mechanical thrombectomy following catheter directed thrombolysis combined with a continuous intra-arterial infusion of low-dose tirofiban for ACIST. Follow-up angiography 9 months after stenting showed lessens restenosis and no in-stent thrombosis. Carotid thrombosis after CAS can be resolved by mechanical thrombectomy combined with in-stent catheter directed intra-arterial thrombolysis with a continuous infusion of low-dose tirofiban for more than $24 \mathrm{hr}$ and subsequent treatment with antithrombotic agents. This management is a feasible solution to ACIST.
\end{abstract}

Keywords: Acute Carotid In-Stent Thrombosis (ACIST), Mechanical Thrombectomy, Intra-arterial Continuous Thrombolysis, Low Dose Tirofiban

\section{Introduction}

Carotid artery stenting (CAS) shows potential as an effective and safe treatment to alleviate internal carotid artery (ICA) stenosis and prevent future ischemic stroke as well as the need for carotid endarterectomy (CEA) [1]. Various clinical trials are in progress to establish the indications for CAS [2, 3]. Carotid stent thrombosis is a relatively rare complication [4-6], observed in only one of 146 procedures [7]. The reported incidence of carotid thrombosis after CAS ranges from $0.04 \%$ to $2 \%$ [6-8]. However, carotid thrombosis can cause severe neurological deficits. The standard strategy for treatment of acute carotid in-stent thrombosis (ACIST) has not been established, the outcome after recanalization remains unclear. We have performed CAS for 206 patients with ICA stenosis in our hospital using various protection devices since 2010 . Here we describe a case of CAS complicated with stent thrombosis treated by mechanical thrombectomy following in-stent catheter directed thrombolysis.

\section{Case presentation}

A 65-year-old female was admitted to our hospital with recurrent transient right hemiparesis and motor aphasia (TIAs). Computed tomography (CT) on admission did not reveal any abnormal density areas (Fig.1A). T1 and T2 weighted, diffusion weighted magnetic resonance imaging (MRI) showed a lacunar infarction area at the watershed territory of the ICA (Fig.1BCD). Digital Subtraction Angiography (DSA) revealed severe stenosis in the left carotid artery (Fig. 2A). Aspirin (100 mg) and clopidogrel $(75 \mathrm{mg}$ ) were administered daily to maintain the activated partial thromboplastin time between 40 and 60 seconds. However, the patient developed progressive right hemiparesis and motor aphasia 3 days after admission. CT reveals a small low density area at the watershed territory of the ICA (Fig.1E). Emergent angiography showed progressive stenosis with subtotal occlusion (Fig.2B). CAS with distal protection filter was performed to alleviate the ischemia under local anesthesia and 3000 units of heparin were injected intravenously. First, predilation was performed with a $5 \times 30 \mathrm{~mm}$ balloon (Viatrac 14 Plus; Abbott Vascular) at $14 \mathrm{~atm}$. and under distal protection of the Spider

This article is published under the terms of the Creative Commons Attribution License 4.0 Author(s) retain the copyright of this article. Publication rights with Alkhaer Publications. Published at: http://www.ijsciences.com/pub/issue/2019-05/

DOI: 10.18483/ijSci.2023; Online ISSN: 2305-3925; Print ISSN: 2410-4477 
RXTM Embolic Protection Device (EV3. Inc., USA). A Protégé $\AA$ Rx taper stent $(8 \times 40 \mathrm{~mm}$; EV3.) was then placed. After withdrawing the stent delivery system and the cerebral protection filter, angiography performed immediately after CAS revealed sufficient dilation without thrombus (Fig.2CD). The postprocedural angiogram showed the stent looked good. The patient presented no neurologic deficit after the procedure. Salt solution was administered intravenously for 3 hours, without continuous antiplatelet therapy. No TIAs were observed after the treatment.

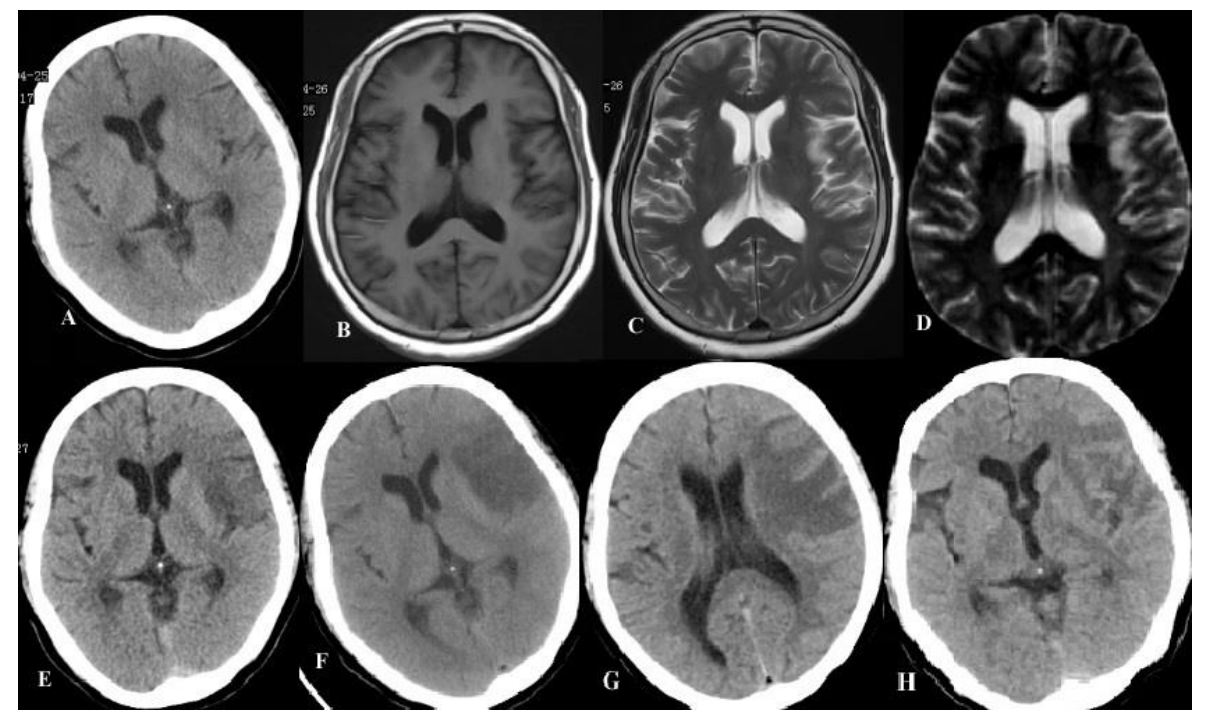

Fig.1 CT and MRI findings in patients with disease. A showing normal head CT after TIA. B, C and D showing T1 and T2 weighted, diffusion weighted magnetic resonance imaging signals at the watershed territory of the ICA on admission. E showed a small low density area at the watershed territory of the ICA before CAS. F showing left temporal-parietal lobe, insular lobe acute cerebral infarction befor recanalization. G revealing a large low density area at the basal ganglia region and watershed zone 2 days after recanalization. $H$ showing left frontotemporal-parietal lobe, left insular lobe infarction area was smaller than before 12 days after recanalization.

Unfortunately, three hours after CAS, the patient developed progressive right hemiparesis and motor aphasia again. CT did reveal an expanded low density area (Fig.1F). Emergent angiography revealed stent occlusion due to acute carotid in-stent thrombosis (ACIST) (Fig.2E). The distal ICA was also occluded due to the giant thrombus formation. We decided to attempt in-stent local intra-arterial contact thrombolysis with high-dose tirofiban to recanalize the occluded ICA. An Excelsior xt 27 microcatheter (Stryker, Fremont, California) was navigated distal to the thrombus over a Synchro 0.014 microwire (Stryker, Fremont, California) and positioned to the in-stent thrombosis and a total dose of $2.5 \mathrm{mg}$ of tirofiban was injected slowly into the thrombus via the microcatheter within $10 \mathrm{~min}$, which led no recanalization. When the procedure was finished 5 min later, angiography revealed that the ICA was still occluded (Fig.2F).

Then, we decided to attempt rheolytic mechanical thrombectomy to recanalize the occluded ICA. First, the same microguidewire was easily introduced into the distal ICA beyond the thrombus to the M1 segment of the left middle cerebral artery. Then, the same microcatheter was gently advanced over the microguidewire, passing beyond the thrombosis. Angiography using the microcatheter confirmed that the thrombus did not extend to the distal M2 segment of the left middle cerebral artery beyond the distal edge of the occlusion (Fig.2G). Next, mechanical thrombectomy was to place Solitaire $(6 \times 30 \mathrm{~mm}$, ev3 Inc., Irvine, California) in the thrombus for 5 minutes and then pull it out. After withdrawing the Solitaire, angiography revealed recanalization of the occluded ICA (Fig.2HI).

Thus, the procedure was finished without additional stent placement in stent. Unfortunately, there still some thrombi were detected in the tent because of the emergent procedure. The neurological deficits partially improved immediately after mechanical thrombectomy. Thus, it needs to follow catheter directed thrombolytic combined with a continuous intra-arterial infusion of $150 \mu \mathrm{g} / \mathrm{hr}$ (the low-dose is $0.1 \mu \mathrm{g} / \mathrm{kg} / \mathrm{min}$ and high-dose is $0.4 \mu \mathrm{g} / \mathrm{kg} / \mathrm{min}$ according to the instruction manual) tirofiban (Tirofiban Hydrochloride for Injection, Shandong new times Pharmaceutical Co., Ltd. China) for the ACIST (Fig.2J). This procedure was finished 26 hours later and subsequent treatment with antithrombotic agents. 


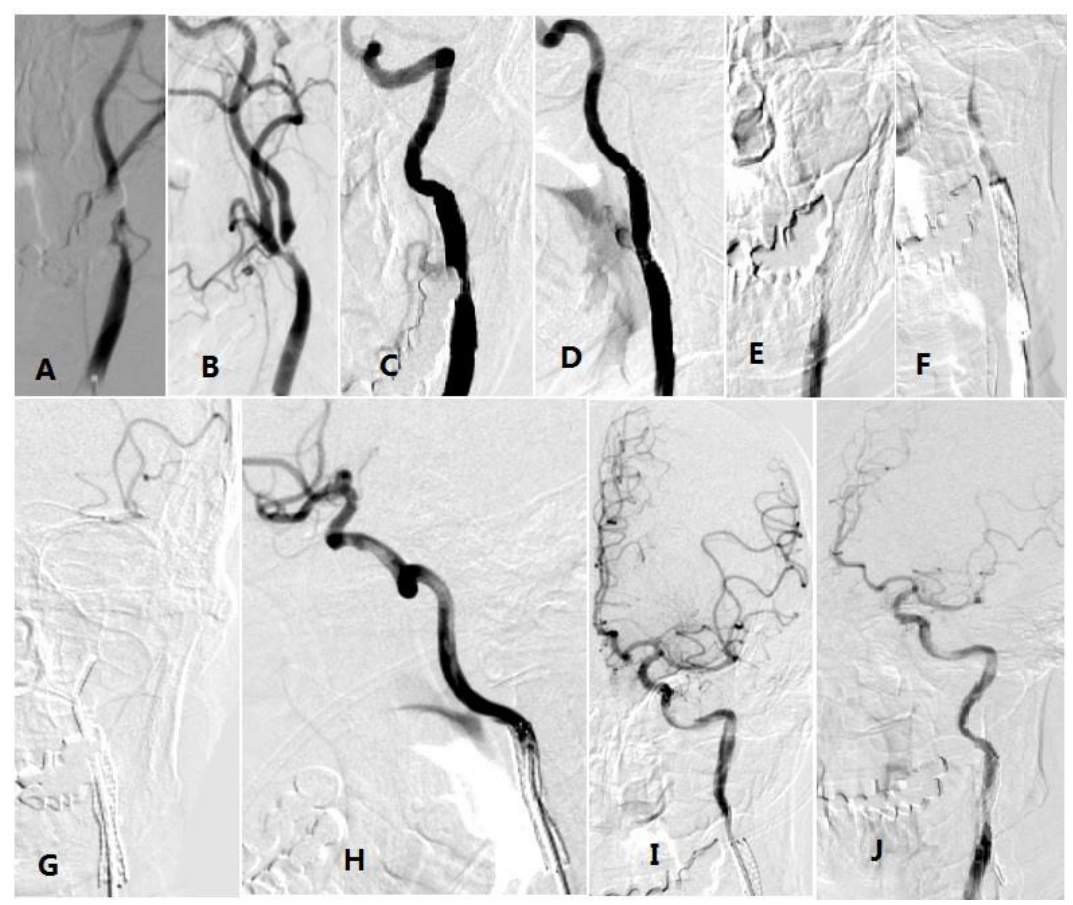

Fig.2 DSA findings of left carotid artery before and after operation. A and B revealed severe left internal carotid artery stenosis with subtotal occlusion thrombus before operation. $\mathrm{C}$ and D revealed sufficient dilation without thrombus in left internal carotid artery after CAS. E and F showed total occlusion due to in-stent thrombosis. G showed patency of distal M2 segment of left middle cerebral artery. H and I showed recanalization of left internal carotid artery after rheolytic mechanical thrombectomy. $\mathrm{H}$ showed catheter directed thrombolytic combined with a continuous intra-arterial infusion of low-dose tirofiban $(0.15 \mathrm{mg} /$ hour $)$ for the ACIST. The tube was placed in the stent.

CT reveals a large low density area at the basal ganglia region and watershed zone (Fig.1G) 2 days after recanalization. Follow-up angiography 1 week after recanalization showed reduced thrombus. Ultrasonography showed no floating thrombus around the stent. Ultrasonography 10 days after CAS showed that the stent was patent without remarkable thrombus. However, Computed tomography angiography (CTA) showed 60\% restenosis in left internal carotid artery 12 days after recanalization (Fig.3A) and the infarct size of the left frontotemporal parietal lobe and the left insula was narrower than before (Fig. $1 \mathrm{H})$. The patient was discharged with mild right hemiparesis 14 days after the CAS procedure. Follow-up ultrasonography 1 month after discharge did not show any thrombus. The patient's hemiparesis disappeared. Follow-up CTA revealed only $10 \%$ restenosis 9 months after stenting (Fig.3B). The patient had not developed any new neurological deficits. Her progress has been closely observed continuing two antiplatelet agents without further treatment.

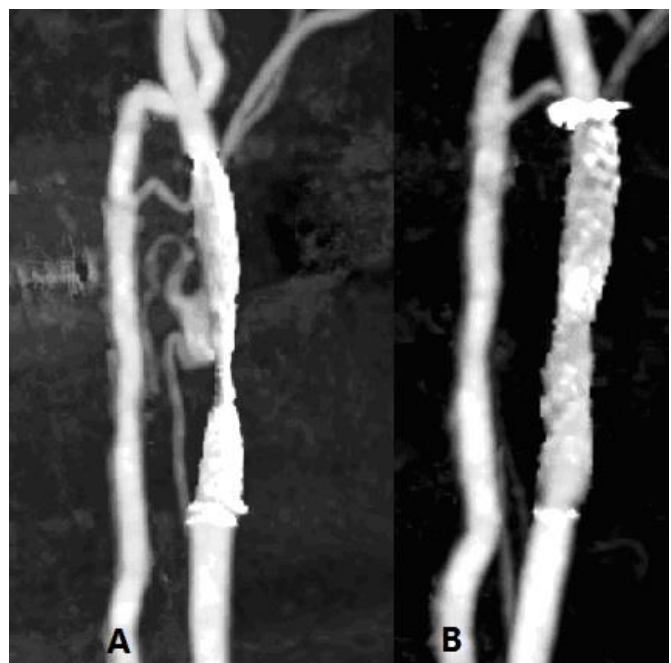

Fig.3 Follow-up CT angiography of left carotid artery after recanalization. A: CT angiography showed $60 \%$ restenosis in left internal carotid artery 12 days after recanalization. B: Follow-up CT angiography revealed only $10 \%$ restenosis 9 months after stenting.

\section{Discussion}

Carotid artery stenting (CAS) is widely performed for extracranial carotid artery stenosis. It was regarded as a relatively safe, less invasive treatment of internal carotid artery stenosis. Acute carotid in-stent thrombosis (ACIST) is a rare but has devastating effects on the survival of the patient. A rapid 
diagnosis and prompt recanalization of the internal carotid artery are needed to minimize the ischemic insult and the reperfusion injury. Although the standard strategy for treatment of carotid thrombosis has been established [9], and the outcome after successful recanalization is clear; but the standard strategy for treatment of ACIST has not been established and the outcome after treatment remains unclear. According to the current literature on this devastating complication of CAS, the earlier treatment was surgical recanalization[10], and the subsequent treatments are increasing. For ACIST when the stent does not fully adhere to the blood vessel, a mechanical approach should be a feasible solution to the problem. $\mathrm{Hu} \mathrm{W}$. at al [11] used a protection filter deployed at the distal part of the stent and redilation of the stent performed with a balloon. Thus, carotid revascularization was performed successfully, proven by postprocedural angiogram. In addition to this management, in conjunction with the intravenous administration of tissue plasminogen activator, performed additional stent placement on the stented portion of the ICA and also gained full recanalization of the ICA [12].

Although many factors can lead to ACIST, so far, no closely related etiologies have been found [13]. According to our study discontinuation of antiplatelet therapy, resistance to antiplatelet agents and inherent or acquired thrombotic disorders are the main causes of thrombosis. For our case, the patient was in a state of hypercoagulability. Treatments of hypercoagulable patients, Miyakoshi et al. [14-15] demonstrated to us that controlled anticoagulation therapy can be an effective treatment for cases in which a thrombus recurs within a stent after CAS evaluates the safety and efficiency of low dose intra-arterial tirofiban $(150 \mu \mathrm{g} / \mathrm{hr})$ during mechanical thrombectomy of acute ischemic stroke (AIS) patients to facilitate the reperfusion of distal vessel. They concluded that patients treated with tirofiban presented lower mortality when compared with those who were not treated with tirofiban without significant difference. Intra-arterial tirofiban may be safe in mechanical thrombectomy of AIS to facilitate the reperfusion of distal vessel, but has no beneficial effect on prognosis.

To achieve a higher recanalization rate for mechanical Solitaire thrombectomy, we used an intra-arterial low-dose tirofiban $(150 \mu \mathrm{g} / \mathrm{hr})$ injection into the occluded segment after mechanical Solitaire thrombectomy lasting for 26 hours, a disastrous outcome was averted. To the best of our knowledge, this patient is the first reported case of successful recanalization by mechanical thrombectomy and following in-stent catheter-directed thrombolysis with a continuous intra-arterial infusion of low-dose tirofiban for ACIST. The patient had large hemispheric stroke and recovered shortly thereafter. For ACIST when the patient is in a state of hypercoagulability, a mechanical approach follow-up continuously intra-arterial thrombolysis should be a feasible solution to the problem. As a result of this combined method of clot removal, a disastrous outcome was averted and resolution of neurological symptoms.

\section{Reference}

1. Jalbert JJ, Nguyen LL, Gerhard-Herman MD, et al. Comparative Effectiveness of Carotid Artery Stenting Versus Carotid Endarterectomy Among Medicare Beneficiaries. Circ Cardiovasc Qual Outcomes. 2016 May; 9(3):275-85.

2. Wholey $\mathrm{MH}$, Wholey $\mathrm{M}$, Mathias $\mathrm{K}$, et al. Global experience in cervical carotid artery stent placement. Catheter Cardiovasc Interv. 2000 Jun; 50(2):160-7.

3. Powers WJ, Rabinstein AA, Ackerson T, et al. 2018 Guidelines for the Early Management of Patients With Acute Ischemic Stroke: A Guideline for Healthcare Professionals From the American Heart Association/American Stroke Association. Stroke. 2018 Mar; 49(3):e138.

4. Bush RL, Bhama JK, Lin PH, et al. Transient ischemic attack due to early carotid stent thrombosis: successful rescue with rheolytic thrombectomy and systemic abciximab. J Endovasc Ther. 2003 Oct; 10(5):870-4.

5. Terada $\mathrm{T}$, Tsuura $\mathrm{M}$, Matsumoto $\mathrm{H}$, et al. Results of endovascular treatment of internal carotid artery stenoses with a newly developed balloon protection catheter. Neurosurgery. 2003 Sep; 53(3):617-25.

6. Moulakakis KG, Mylonas SN, Lazaris A, et al. Acute Carotid Stent Thrombosis: Comprehensive Review. Send to Vasc Endovascular Surg. 2016 Oct; 50(7):511-521.

7. Roubin GS, New G, Iyer SS, et al. mmediate and late clinical outcomes of carotid artery stenting in patients with symptomatic and asymptomatic carotid artery stenosis: a 5-year prospective analysis. Circulation. 2001 Jan 30;103(4):532-7.

8. New G, Roubin GS, Iyer SS, et al. Carotid artery stenting: rationale, indications, and results. Compr Ther. 1999 Aug-Oct; 25(8-10):438-45.

9. Powers WJ, Rabinstein AA, Ackerson T, et al. 2018 Guidelines for the Early Management of Patients With Acute Ischemic Stroke: A Guideline for Healthcare Professionals From the American Heart Association/American Stroke Association., Stroke. 2018 Mar;49(3):e138.

10. Markatis F, Petrosyan A, Abdulamit T, et al. Acute carotid stent thrombosis: a case of surgical revascularization and review of treatment options. Vascular 2012; 20: 217-220.

11. Hu W, Wang L, Wang G. Acute In-Stent Thrombosis after Carotid Angioplasty and Stenting: A Case Report and Literature Review. Interv Neurol. 2018 7(5):265-270.

12. Kurisu K, Manabe $\mathrm{H}$, Ihara T. Case of symptomatic subacute in-stent thrombosis after carotid angioplasty and stenting for severe carotid stenosis . No Shinkei Geka, 2007, 35(10):1001-1005.

13. Ozaki S, Tagawa M, Matsumoto S, et al. Pathogenesis of in-stent thrombosis after carotid artery stenting. No Shinkei Geka, 2014 Nov; 42(11):1009-17.

14. Miyakoshi A, Toda $\mathrm{H}$, Hayase $\mathrm{M}$, et al.Anticoagulant therapy for recurrent in-stent thrombosis following carotid artery stenting: A case report. Interv Neuroradiol. 2017 Oct; 23(5):521-526.

15. Yu T, Lin Y, Jin A, et al. Safety and Efficiency of Low Dose Intra-arterial Tirofiban in Mechanical Thrombectomy During Acute Ischemic Stroke. Curr Neurovasc Res. 2018; 15(2):145-150. 